\title{
New concepts in Perioperative Fluid Management
}

R.K.Kamel, E.A.Shaboob, and G.S.W.Youssef

Anesthesiology and Intensive care, Dept., Faculty of Medicine, Benha Univ., Benha, Egypt

E-mail: Gena Shahat@yahoo.com

\begin{abstract}
Background: Perioperative fluid management is an essential concern during the whole procedure and effective management of the fluid should be seen as accontinuation over the full hospital intake of the patient. This research sought to examine current studies in the perioperative fluid adjustment in order to preserve and recover tissue fluid and electrolyte hemeostasis and central euvolemia. Methods: The research was carried out in the same date period in MEDLINE, Embase, Pubmed and CINAHL Plus, using the following media terms: 'Perioperative; Fluid Management; Intensive Care; Anesthesia' including articles 2000 to 2020, Results and findings: Liberal fluids of up to 20 or 30 $\mathrm{ml} / \mathrm{kg} / \mathrm{h}$ may have some advantages for ambulatory patients (such as decrease in post-operative sleepiness, nausea, and discomfort), and international guidance recommends maintenance fluids of $1-2 \mathrm{ml} / \mathrm{kg} / \mathrm{h}$ for lengthier or longer major procedures. The fluid challenge remains one of the most essential instruments for an anesthetician to evaluate the fluid response. When the patient is fluid depleted and tolerant of additional fluids, a little but fast fluid bolus should raise pre-load enough to induce a significant rise in stroke volume and cardiac output. Fluid management via improved restoration paths or a goal-oriented strategy has shown both to decrease post-operative problems.
\end{abstract}

Keywords: Perioperative, Fluid Management, Intensive care, Anesthesia.

\section{Introduction}

Water accounts for about $60 \%$ of typical adult body weight, depending with age, gender and body composition. Adipose tissue contains little water in comparison with other tissues, leading to a marked variability in body proportions between 75 percent in adipose and 45 percent in adipose, the total body weight is divided between body anatomic and functional fluid compartments, the major division between intracellular fluid and extracellular fluid.

Perioperative fluid management is an essential concern during the whole procedure and the optimum management of the fluid should be considered as acontinuation through the full admission of the patient. The overall objective of preoperative fluid management is to avoid dehydration of patients even before the operation begins. Clearly, maintaining an intraoperative azero balancing strategy is considerably simpler if patients begin operations in an abnormal euvolemic condition [2].

The principal intraoperative objectives are simply to replace continuous losses without extra salts and water and to ensure that patients have pure maintenance needs. The resumption of regular oral food and fluids should be encouraged as early as feasible and fluids must cease as soon as they can, and additional needs may relate to postoperative problems such as bleeding and severe septic sepsis[3].

The choice of the intravenous fluid to be used is also essential for a successful improved recovery.

Generally speaking. All intravenous fluids are classified in just three classes: Crystalloid, Colloid and Blood produt.

Cristalline solutions, for example, are combined with electroliyte or glucose ions, and sodium cholride ions combine water to produce saline solutions. They are best used to replace insensitive losses (typically combined with electrolyte dispensing, for example. Sweating causes loss of salt and water) Some may also be employed as resuscitative liquids since they can affect hemodynamic state, the exceptions being dextrose-based solutions because cell glucoseuptake is so quick that there are no substantial hemodynamic effects [4].

Colloid solutions, instead of electrolytic ions, are solutions combined with macro-molecular solutes. Examples include solutions such as starch, gelatin or dextran. These solutes exert osmotic pressure across the wall of glycocalyx endothelia and are believed to stay longer in the intravascular region (thereby exerting a prolonged hemodynamic impact than crystalloid solutions) owing to their particle size. [5]

Blood products consist of different blood components, including red cells, platelets, fresh frozen plasma (FFP) or mixtures of coagulation factor. [5].

The fluid challenge remains one of the most essential instruments for an anesthetician to evaluate the fluid response. If the patient is fluid depletion and tolerable for additional fluids, a slim but fast fluid bolus should raise preload enough to produce a detectable rise in the volume of a stroke and in cardiac output.

Initially liberal Fluid Therapy was encouraged to maintain approposed third space in a perioperative way, as evidence to support this theoretical compartment has always been fearful, and our understanding of the damage to glycocalyx has increased to amorous and restrictive fluid administration, resulting in an interstitial oedema. [6] Fluid management through improved recovery pathways or via a goal-oriented strategy both have shown reduction of post-operational problems. Agoaldirected fluid approach can also add extra benefits to improved recovery protocols especially in patients at great risk. The greatest benefit from a pre-operative approach is nearly the presence of patients who have not successfully optimised their pre-op fluid status and start an operation-responsive fluid. [7] 
The UK guidelines advised adopting a personalised fluid regimen for all enhanced recovery patients using azero balance approach[7].

This research examines current studies on fluid adjustment during the perioperative phase to preserve and recover fluid tissue and hemeostasis and central euvolemia electrolytes.

\section{Methods}

This is a review article, The search was performed in MEDLINE, Embase, Pubmed and CINAHL Plus in the same date range with the following mediacl terms: "Perioperative; Fluid Management; Intensive care; Anesthesia",including articles from 2000 to 2020, Excluded articles from review are those of language other than English

\section{Results}

Enhanced recovery pathways offer significant benefits to patients in terms of reducing morbidity and also length of stay after elective surgery, and they are gradually being used in more and more surgical specialties [8].

Table 1[8] provides requirements for a typical enhanced recovery pathway under the UK Enhanced Recovery Partnership Programme.

Fluid therapy is expressly mentioned (and emphasised) in each of the three stages of the table (preoperatively, intraoperatively, as well as postoperatively).

It is now widely recognised that better recovery pathways may safely reduce hospital stay, but what are the most important components for achieving this in the enhanced recovery plan remains unchallenged. Although a recent exhaustive study has not shown that C-reactive protein (CRP) levels are changed by fluid therapy () Since fluid management varies considerably from centre to centre, and various fluid protocols affect the incidence of surgical complication (nearly $50 \%$ ), it is fair to suggest that the optimum peripheral fluid management is an important component of any better rehabilitation protocol [9].
Fluid Therapy in Enhanced Recovery: A "ZeroBalance" Approach Perioperative fluid management is an important consideration throughout the whole surgical pathway, and optimal fluid management should be viewed as a continuum throughout the patient's whole hospital admission. Suboptimal management at any point will not only lead to significantly longer hospital admissions, but also risks compromising the benefits conferred by other elements of the enhanced recovery package [7].

The overarching focus as with many enhanced recovery elements should be to always aim for as near physiological normality as possible. In the context of fluids, this can be thought of as avoiding dehydration and hypovolemia or fluid overload with their associated complications. Inadequate fluid administration results in insufficient perfusion pressures, reducing oxygen delivery and increasing anaerobic metabolism, which ultimately leads to cell death and end-organ failure [10].

Conversely, excess fluid administration can have equally harmful consequences, raising hydrostatic pressures and increasing levels of atrial-natriuretic peptides, which damage the delicate glycocalyx layer of the vascular endothelium.This renders blood vessels "leaky" and causes damaging tissue edema to develop in the interstitium, which again impairs tissue and organ oxygenation endothelium, This interstitial edema, together with high salt loads from excess crystalloid infusion, can also lead to postoperative ileus and further increase patient's length of stay [10].

"Zero-balance" is also the term that the American Society for Enhanced Recovery (ASER) has adopted in their guidelines on perioperative fluid management to encourage this approach, Currently, consensus UK guidelines recommend using an individualized fluid plan with a zero-balance approach in all enhanced recovery patients. They also emphasize that some patients will benefit from cardiac output optimization through a goal-directed approach, with higher risk patients having higher risk operations most likely to gain [7].

Table (1) Components of a typical enhanced recovery pathway.

\begin{tabular}{|c|c|c|}
\hline \multicolumn{3}{|c|}{ Enhanced recovery elements as suggested by the UK Enhanced recovery Partnership Programme } \\
\hline Preoperative & Intraoperative & Postoperative \\
\hline Pre -op visit & Antibiotics prior to first incision & Nasogastric tube removal \\
\hline Patient assessed for surgery & Epidural or regional analgesia & Avoid crystalloid over load \\
\hline $\begin{array}{l}\text { Patient explanation for } \\
\text { enhanced recovery }\end{array}$ & $\begin{array}{l}\text { Use fluid management } \\
\text { technologies to individualize } \\
\text { fluid therapy }\end{array}$ & $\begin{array}{l}\text { Use agoal directed style of fluid } \\
\text { management }\end{array}$ \\
\hline $\begin{array}{l}\text { Education given (eg,therapy in } \\
\text { MSK or stoma in colorectal }\end{array}$ & Avoid excess crystalloids & $\begin{array}{l}\text { Post op nutrition (encourage } \\
\text { early oral intake ) }\end{array}$ \\
\hline Oral bowel prep avoided & Hypothermia avoidance & Nausea and vomiting control \\
\hline Admitted on day of surgery & Avoid abdominal drains & Early mobilization \\
\hline Carbogydrate drinks given & & Early removal of catheter \\
\hline Maintain good pre - op & & Avoid systemic opoids \\
\hline hydration & & \\
\hline Avoidance of sedatives & & \\
\hline
\end{tabular}




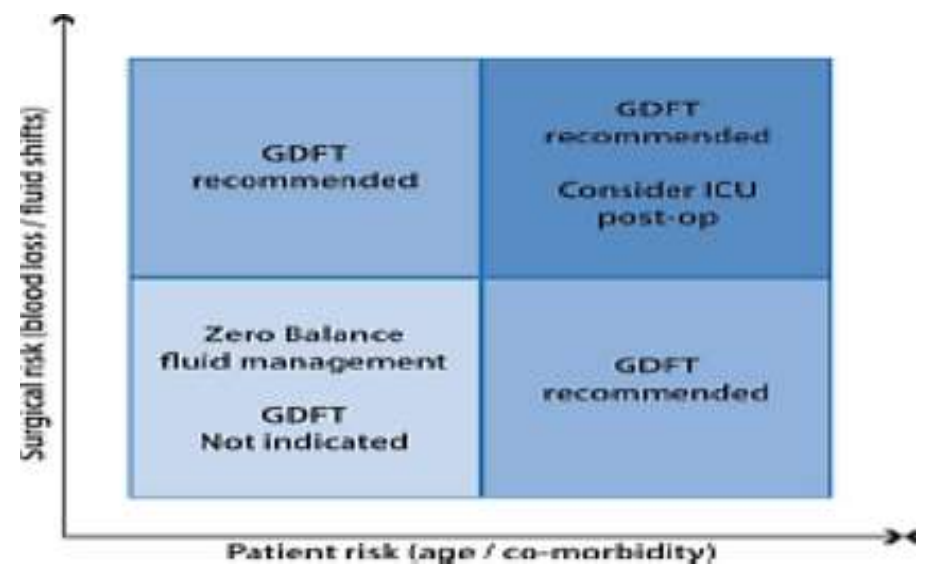

Fig. (1) The fluid management approach used should depend on both patient and surgical risk factors, with goaldirected fluid therapy (GDFT) indicated in higher risk cases

The UK Enhanced Recovery Consensus statement specifically recommends the use of intraoperative fluid management technology (such as the esophageal Doppler) in any operation, with any of the following features [11].

- Major surgery with a 30-day mortality rate of $>1$ $\%$

- Major surgery with anticipated blood loss of $>500$ $\mathrm{ml}$

- Major intra-abdominal surgery

- Intermediate surgery (30-day mortality $>0.5 \%$ ) in high-risk patients (e.g., age $>80$, history of left ventricular failure or previous ischemic heart disease or stroke)

- Unexpected blood loss requiring >2 1 of fl uid replacement

- Patients with evidence of ongoing hypovolemia or tissue hypoperfusion (e.g., persistent lactic acidosis)

Fluid Choice

Choosing which intravenous fluid to use is also vitally important to a successful enhanced recovery pathway. In general, all intravenous fluids fall into one of just three categories: Crystalloids, Colloids and blood products

Which fluid type is the "best type" of fluid remains hotly debated. Ideally,fluid losses should be replaced with fluids with a similar composition in an aim to keep physiological normality [10].

For example, blood loss should be replaced with blood products wherever possible, such as packed red cells as well as with platelets and other clotting factors if the blood loss is significant.

Insensible losses (such as through perspiration and respiration) should be replaced with balanced crystalloid solutions, and $0.9 \%$ saline solutions (including some colloids that are mixed with $0.9 \%$ solutions) should be avoided wherever possible. There are very few studies that show their administration to result in clinical benefit, and they have frequently been shown to cause a hyperchloremic acidosis through excess sodium and chloride administration, which may be harmful [7].
The British Consensus Guidelines on Intravenous Fluid Therapy for Adult Surgical Patients (GIFTASUP) advise that patients receive the following to meet their minimum daily maintenance requirements.

- 1-2 $\mathrm{mmol} / \mathrm{kg}$ of sodium

- $1 \mathrm{mmol} / \mathrm{kg}$ of potassium

- $30 \mathrm{ml} / \mathrm{kg}$ water

Consequently, maintenance fluid regimes should aim to replace the above, and ideally at a rate of less than $2 \mathrm{ml} / \mathrm{kg} / \mathrm{h}$ (including any drug infusions) according to the consensus statement from the British Enhanced Recovery Partnership.

Where intravenous fluids do need to be continued postoperatively, these guidelines strongly recommend using a low-sodium crystalloid solution (e.g., $0.18 \%$ sodium $/ 4 \%$ dextrose with potassium) to minimize the risk of developing postoperative ileus from excessive sodium administration [11].

In terms of replacing other volume losses, most goal-directed fluid studies have used colloid boluses. This is because colloid boluses are thought to increase stroke volume and blood pressure more (and also more quickly) than the same volume of a crystalloid solution, due to colloids being less likely to leak across the glycocalyx and out of intravascular space as rapidly as crystalloid solutions [7].

The Colloids Versus Crystalloids for the Resuscitation of the Critically Ill (CRISTAL) trial (a large, multicenter randomized control trial comparing crystalloids and colloids for resuscitation of hypovolemic shock) showed a significant reduction in 90-day mortality in the colloid group, suggesting benefit in using colloid boluses in fluid-responsive patients to replace volume loss [12]

However, at least two other large randomized trials have recently suggested that using starch based fluids in critical care patients is associated with an increased risk of kidney injury or the need for renal replacement therapy, throwing this perceived survival benefit into question [13]. 


\section{Discussion}

The primary goal of preoperative fluid management is to avoid the dehydration of patients before the procedure begins. Clearly, if the patient begins operations in a normal euvolemic condition, it is more simpler to maintain an approach of zero balance intraoperatively [7].

Although patients obviously need to forgo solid meals for choicing operation in order to reduce the danger of aspiration, worldwide recommendations increasingly recognise the significance of not extending the fasting time for fluids more than 2 hours before surgery. Cochrane tests indicate that consuming clear liquids up to 2 hours before to an operation is not linked with an increased risk of aspiration or other problems in adults or children. These studies show, however, that consuming clear fluids decreased adult stomach volumes and made both adults' and their preoperative experiences more pleasant. In its recommendations, the European Society of Anaesthesiology also advises adults and children to consume liquids up to 2 hours in advance[14].

For similar reasons, many improved rehabilitation regimens also support the avoidance of artificial bowel preparation in many patients, although this becomes more contentious. Mechanical bowel preparations showed that dehydration has improved and patient comfort has decreased, without decreasing the risk of early postoperative problems in most cases[15].

In addition to being preoperatively hydrated, the nutritional condition of patients should also be improved with carbohydrate energy drinks before surgery. These beverages also reduce patient pain in the course of waiting for surgery and decrease insulinresistance postoperatively due to increased insulin activity. Depending on the nutrition content, they may safely be consumed 2-3 hours before the operation.

Fluid Management Intraoperative Again, the primary objectives of an intraoperative fluid balance in enhanced recovery pathways should be to preserve physiological normalcy, that means, eupholism should be maintained and electrolyte disruption minimised, as with most other enhanced recovery components. Successful preoperative fluid management should enable patients to undergo surgery adequately hydrated and therefore replenish continuing losses without providing extra water and salt[7] as the primary intraoperative objectives.

Insensible losses (e.g. suction or urine) constitute a relatively low proportion of continuing losses, which are frequently replaced with crystalloids by a maintenance fluid system. This was usually shown to be less than $1 \mathrm{ml} / \mathrm{kg} / \mathrm{h}$ under normal conditions in a direct measurement of intraoperative evaporative losses and it is important to remember that providing fluids exceeding this rate can lead to harm and postoperative complications (such as ileus as explained earlier) rapidly[3].

In ambulatory medication (for example, decreasing postoperative drowsiness, nausea and pain), the liberal fluid administration up to $20-30 \mathrm{ml} / \mathrm{kg} / \mathrm{h}$ may offer some benefit, International guidelines suggest that maintenance fluids should be administered at $1-2 \mathrm{ml} / \mathrm{kg} / \mathrm{h}$ for all longer or greater operations[11].

However, most intraoperative losses are intravascular losses in volume. The patient may lose volume, for example, via blood loss or compartmental fluid changes, such as the development of interstitial edoema, due to the inflammatory surgical response. These losses will need the replacement of comparable amounts of similar fluids. In the case of severe cante haemorrhage, for example, the blood loss should preferably be replaced by blood products, including platelets and clotting factors.

Postoperatively, patients should be encouraged, and IV fluids should be discontinued as soon as feasible, to resume normal oral meals and fluids in improved recovery pathways as early as possible. The likelihood of the development of postoperative ileus is increased further by continuing intravenous fluid in a postoperative period, especially since the capacity of patients to excrete and eliminate both salt and chloride is decreased postoperatively [10].

For this reason, when fluids must continue after the operation, low volume fluids with a relatively low sodium content should be considered especially when excess sodium and chloride are present intraoperative in majority of the patients[7].

The postoperative continuation of intravenous fluid will also adversely affect other improved recovery pathway components. One of the key focuses of improved post-operative recovery is to promote early mobility and patients are less inclined to move if linked with intravenous fluid. In addition, catheters will inhibit mobility in patients and should be removed as soon as possible [7].

Adequate analgesia are also essential for maximising early mobilisation opportunities, although laxatives may also be needed, depending on the operation done, to prevent constipations and urine retention.

Early oral intake is also independent from surgery. A comprehensive study has revealed that the risk of surgical infection decreases substantially during early feeding and also automatically decreases hospital stay. It may also reduce the risk of anastomotic operation of dehiscence, wound infection, pneumonia, intraabdominal abscess and death, but the statistical significance of these has not been achieved in the meta-analysis [16].

Ultimately though, goal-oriented treatment has shown to be a safe operation repeatedly. Better fluid management via improved recovery routes or goals was both proven to decrease postoperative complications on an independent basis. An targetbased fluid strategy may also provide additional benefit to improved recovery procedures, especially in patients at greater risk, but a large, high-performance clinical study is need to respond conclusively[17]. 
The main advantage of a goal-directed strategy is virtually likely in patients who have not effectively adjusted their preoperative fluid status and will start surgery with fluid responsiveness. Since it is impossible to anticipate which patients will fall into this category, one idea is to employ target-driven treatment in all patients to guarantee that the patients who benefit from target-driven fluid therapy still get therapy [7].

When substantial volumes of blood loss or significant cross-cutting fluid changes are anticipated for particular procedures, it appears reasonable to view fluid therapies based on cardiac output monitoring as best practice[18].

\section{Conclusion}

Perioperative fluid management is an essential part of the whole procedure and optimum fluid management should be seen as an accontinuation during the entire hospital stay of the patient. The primary goal of preoperative fluid management is to avoid the dehydration of patients before the procedure begins. The re-start of regular oral meals and drinks should be encouraged as quickly as possible, and fluids should cease once they have done so. The restriction of fluid to achieve zero equilibrium is also a crucial component of improved post-operation recovery (ERAS), a guideline that supports early recovery among patients following major surgery. Liberal fluids of up to 20 or $30 \mathrm{ml} / \mathrm{kg} / \mathrm{h}$ may have some advantages for ambulatory patients (such as decrease in post-operative sleepiness, nausea, and discomfort), and international guidance recommends maintenance fluids of $1-2 \mathrm{ml} / \mathrm{kg} / \mathrm{h}$ for lengthier or longer major procedures. The fluid challenge remains one of the most essential instruments for an anesthetician to evaluate the fluid response. When the patient is fluid depleted and tolerant of additional fluids, a little but fast fluid bolus should raise pre-load enough to induce a significant rise in stroke volume and cardiac output. Fluid management via improved recovery pathways or an objective method have both shown a reduction in postoperative problems

\section{References}

[1] C.M.M.Prado. "The association between body composition and toxicities from the combination of Doxil and trabectedin in patients with advanced relapsed ovarian cancer," Appl. Physiol. Nutr. Metab., vol. 39, pp. 693-698, 2014.

[2] J.P.Miller, A.-S.Lambert, W.A.Shapiro, I.A.Russell, N.B.Schiller, and M.K.Cahalan, "The adequacy of basic intraoperative transesophageal echocardiography performed by experienced anesthesiologists," Anesth. Analg., vol. 92, pp. 1103-1110, 2001.

[3] D.N.Lobo, K.A.Bostock, K.R.Neal, A. C. Perkins, B. J. Rowlands, and S. P. Allison, "Effect of salt and water balance on recovery of gastrointestinal function after elective colonic resection: a randomised controlled trial," Lancet, vol. 359, pp. 1812-1818, 2002.

[4] V.A.Bennett and M.Cecconi, "Perioperative fluid management: From physiology to improving clinical outcomes," Indian J. Anaesth., vol. 61, p. 614, 2017.

[5] L.H.C.Navarro . "Perioperative fluid therapy: a statement from the international Fluid Optimization Group," Perioper. Med., vol. 4, pp. 1-20, 2015.

[6] M.Jacob, D.Chappell, and M. Rehm, "The 'third space'-fact or fiction?," Best Pract. Res. Clin. Anaesthesiol., vol. 23, pp. 145-157, 2009.

[7] T. E. Miller, A. M. Roche, and M. Mythen, "Fluid management and goal-directed therapy as an adjunct to Enhanced Recovery After Surgery (ERAS)," Can. J. Anesth. Can. d'anesthésie, vol. 62, pp. 158-168, 2015.

[8] J. C. Simpson et al., "Enhanced recovery from surgery in the UK: an audit of the enhanced recovery partnership programme 2009-2012," BJA Br. J. Anaesth., vol. 115, pp. 560-568, 2015.

[9] B. Brandstrup et al., "Effects of intravenous fluid restriction on postoperative complications: comparison of two perioperative fluid regimens: a randomized assessor-blinded multicenter trial," Ann. Surg., vol. 238, pp. 641, 2003.

[10] M.R.Edwards and M.G.Mythen, "Fluid therapy in critical illness," Extrem. Physiol. Med., vol. 3, pp. 1-9, 2014.

[11]M. G. Mythen . "Perioperative fluid management: Consensus statement from the enhanced recovery partnership," Perioper. Med., vol. 1, pp. 1-4, 2012.

[12]D. Annane ."Effects of fluid resuscitation with colloids vs crystalloids on mortality in critically ill patients presenting with hypovolemic shock: the CRISTAL randomized trial," Jama, vol. 310, pp. 1809-1817, 2013.

[13] A. Perner . "Long-term outcomes in patients with severe sepsis randomised to resuscitation with hydroxyethyl starch 130/0.42 or Ringer's acetate," Intensive Care Med., vol. 40, pp. 927934, 2014.

[14]I. Smith . "Perioperative fasting in adults and children: guidelines from the European Society of Anaesthesiology," Eur. J. Anaesthesiol. EJA, vol. 28, pp. 556-569, 2011.

[15] B.Jung, L.Påhlman, P.Nyström, and E. Nilsson, "Multicentre randomized clinical trial of mechanical bowel preparation in elective colonic resection," Br. J. Surg., vol. 94, pp. 689-695, 2007.

[16] S.J.Lewis, M.Egger, P.A.Sylvester, and S. Thomas, "Early enteral feeding versus "nil by mouth' after gastrointestinal surgery: systematic review and meta-analysis of controlled trials," Bmj, vol. 323, pp. 773, 2001. 
[17] C.W.Lai . "Randomized controlled trial of stroke volume optimization during elective major abdominal surgery in patients stratified by aerobic fitness," BJA Br. J. Anaesth., vol. 115, pp. 578-589, 2015.

[18]M. Cannesson ."Assessing the diagnostic accuracy of pulse pressure variations for the prediction of fluid responsiveness: a 'gray zone' approach,” J. Am. Soc. Anesthesiol., vol. 115, pp. 231-241, 2011. 